\title{
Ethical commitment of Spanish oncologists to patients with prostate cancer: reflections on the statements of the new ASTRO/AUA guideline (2019 guideline amendment)
}

\author{
F. Casas ${ }^{1}(1) \cdot$ G. Oses $^{1} \cdot$ K. S. Cortes ${ }^{1} \cdot$ T. D. Barreto ${ }^{1} \cdot$ D. Muñoz $^{1}$
}

Received: 2 July 2019 / Accepted: 9 August 2019 / Published online: 21 August 2019

(c) Federación de Sociedades Españolas de Oncología (FESEO) 2019

A systematic review of the literature was performed in 2013 using PubMed, Embase, and the Cochrane database to identify peer-reviewed publications related to the use of radiation therapy after prostatectomy published from January 1, 1990 to December 15, 2012 [1]. The review yielded 294 articles which had been used to create evidence-based guideline statements. A particular treatment was assigned a rating of A (high-quality evidence), B (moderate-quality evidence), or $\mathrm{C}$ (low-quality evidence) according to the strength of the scientific evidence available, and evidence-based statements of standard and recommendation were developed. The guideline also provides additional guidance as clinical principles (expert opinion) when there was insufficient evidence to rate the treatment. These guideline statements were provided for patient counseling about the use of radiation therapy (RT) in the adjuvant (ART) and salvage (SRT) context, defining biochemical recurrence (BR) and conducting restaging evaluation.

The first amendment to the guideline was made in April 2018 and published in April 2019 [2]. This amendment incorporated evidence from three randomized controlled trials, and a new evidence-based statement was also developed including the uses of hormone therapy (HT) in SRT.

Ten guideline statements (GS) were made in this guideline update: two were standard evidence A, GS 3 and GS 9; three were recommendation and evidence C, GS 5, GS 6 , and GS 7, and the last five were clinical principles (CLP) or expert opinion.

The summary of GS 3, with A as the maximum level of evidence, is that physicians should offer ART in the event of adverse pathologic findings [seminal vesicle invasion (SVI), positive surgical margins $(\mathrm{M}+)$ or extraprostatic extension

$\triangle$ F. Casas

Fcasas@clinic.cat

1 Radiation Oncology Department, Hospital Clínic, Barcelona, Spain
(EE)] because of demonstrated reductions in BR, local recurrence (LR) and clinical progression. The second grade A level of evidence is GS 9 in which clinicians should offer HT to patients treated with SRT with postoperative PSA levels $\geq 0.2 \mathrm{ng} / \mathrm{ml}$.

At the level of recommendation of evidence $\mathrm{C}$, we find GS 5, in which BR is defined as a PSA value after surgery $\geq 0.2 \mathrm{~g} / \mathrm{ml}$; GS 6 which can be considered as an option for restaging evaluation in patients with PSA recurrence; and GS 7 recommends that it is mandatory to offer SRT to patients with PSA or LR after prostatectomy in the absence of metastatic disease.

The expert opinion or CLP is an elaborated consensus between radiation oncologists and urologists and is the most numerous group of statements. In the first GS, specialists emphasize that each patient considered for radical prostatectomy should be informed of the potential adverse pathologic findings associated with a higher risk of cancer recurrence and the possible benefits of additional therapy. In the second GS, physicians agree with the results of three randomized trials which show that patients with SVI, M+, and EE should be informed that ART, compared with prostatectomy alone, reduces the risk of BR, LR and clinical progression. Patients should also be informed that, according to the results of one of these three phase III trials, ART may improve M1 and overall survival (OS) [3]. The other two trials [4, 5] did not demonstrate this benefit, however, they were not statistically designed to identify a significant reduction in M1 or OS with ART. The CLP of GS 4 is that patients should be informed that the development of a PSA recurrence after surgery is associated with a higher risk of development of metastatic prostate cancer or death by the disease. GS 8 emphasizes that patients should be informed that the effectiveness of RT for PSA recurrence is greatest when given at lower PSA levels.

GS 10, the last CLP of the expert panel states that patients should be informed of the possible short- and long-term 
urinary, bowel and sexual side effects of RT as well as the potential benefits of controlling disease recurrence.

This decalogue should inspire the discussion of prostate cancer patients in all uro-oncological tumor boards. These GS have been developed according to evidence-based medicine and with consensus among surgeons, oncologists (urologists) and radiation oncologists who are experts in local prostate cancer treatment. Although medical oncologists are less involved in the localized phase of this cancer, they should not ignore this decalogue, and their collaboration is essential. Their ethical commitment to provide the best care to patients with prostate cancer is the same as that of radiation oncologists and urologists.

Although the interests of each professional are legally binding, the interest of the health of their patients is mandatory. Patients with prostate cancer must know that prostatectomy can cure their prostate cancer, but they must also know that depending on possible pathological factors, they may need other treatments such as postoperative RT and/ or HT to achieve a cure. Patients must also be aware that while prostatectomy may alter sexual potency and urinary continence, postoperative RT may produce further toxicity in addition to these previous complications [6]. Each specialist must explain the possible benefits and secondary effects of the treatments.

The reason for this editorial is to stimulate oncologists (radiation, medical, and surgeon or urologist) to decide whether their multidisciplinary tumor board or prostate cancer protocols based on the previous guideline statements of ART and SRT following prostatectomy published in 1993 are sufficiently incorporated. If this is not so, the incorporation of the new amendment made in April 2019 may be a good opportunity to improve the quality of life, and perhaps, the OS of our patients with prostate cancer.

Acknowledgements We are grateful to Donna Pringle for her English revision of this manuscript.

\section{Compliance with ethical standards}

Conflict of interest None of the authors have any conflicts of interest.
Ethical approval This article does not contain any studies with human participants or animal performed by any of the authors.

Informed consent For this type of study informed consent is not required.

\section{Reference}

1. Valicenti RK, Thompson I Jr, Albertsen P, Davis BJ, Goldenberg SL, Wolf JS, Sartor O, Klein E, Hahn C, Michalski J, Roach M 3rd, Faraday MM, American Society for Radiation Oncology/ American Urological Association. Adjuvant and salvage radiation therapy after prostatectomy: American society for radiation oncology/American urological association guidelines. Int J Radiat Oncol Biol Phys. 2013;86(5):822-8. https://doi.org/10.1016/j. ijrobp.2013.05.029.

2. Pisansky TM, Thompson IM, Valicenti RK, D'Amico AV, Selvarajah $\mathrm{S}$. Adjuvant and salvage radiotherapy after prostatectomy: ASTRO/AUA guideline amendment executive summary 2018. Pract Radiat Oncol. 2019;10:11. https://doi.org/10.1016/j. pro.2019.04.008.

3. Thompson IM, Tangen CM, Paradelo J, et al. Adjuvant radiotherapy for pathologically advanced prostate cancer: a randomized clinical trial. JAMA. 2006;27:2924-35.

4. Bolla M, van Poppel H, Tombal H, et al. Radiation oncology and genitourinary groups. Postoperative radiotherapy after radical prostatectomy for high-risk prostate cancer: long-term results of a randomized controlled trial (EORTC trial 22911). Lancet. 2012;380:2018-27. https://doi.org/10.1016/s0140-6736(12)61253 $-7$.

5. Wiegel T, Bottke D, Steiner U, et al. Phase III postoperative adjuvant radiotherapy after radical prostatectomy compared with radical prostatectomy alone in $\mathrm{pT} 3$ prostate cancer with postoperative undetectable prostate -specific antigen. ARO 96-02/AUO AP 09/95. J Clin Oncol. 2009;27:2924-30.

6. Casas F, Valduvieco I, Oses G, et al. Postoperative adjuvant and very early salvage radiotherapy after prostatectomy in high-risk prostate cancer patients can improve specific and overall survival. Clin Trans Oncol. 2019;21:355-62. https://doi.org/10.1007/s1209 4-018-1931-3.

Publisher's Note Springer Nature remains neutral with regard to jurisdictional claims in published maps and institutional affiliations. 